OPEN ACCESS

Edited by:

Rengyun Liu,

Johns Hopkins University

United States

Reviewed by:

Young Shin Song

Seoul National University Hospital,

South Korea

Salvatore Piscuoglio,

University Hospital of Basel,

Switzerland

*Correspondence:

Mei Yu

yumei_2017@163.com

Specialty section:

This article was submitted to

Cancer Genetics,

a section of the journal

Frontiers in Oncology

Received: 18 October 2018 Accepted: 12 February 2019

Published: 04 March 2019

Citation:

Wang Y, Yu M, Yang J-X, Cao D-Y,

Zhang $Y$, Zhou H-M, Yuan Z and

Shen K (2019) Genomic Comparison

of Endometrioid Endometrial

Carcinoma and Its Precancerous

Lesions in Chinese Patients by

High-Depth Next Generation

Sequencing. Front. Oncol. 9:123.

doi: 10.3389/fonc.2019.00123

\section{Genomic Comparison of Endometrioid Endometrial Carcinoma and Its Precancerous Lesions in Chinese Patients by High-Depth Next Generation Sequencing} Yao Wang, Mei Yu*, Jia-Xin Yang, Dong-Yan Cao, Ying Zhang, Hui-Mei Zhou, Zhen Yuan
and Keng Shen

Department of Obstetrics and Gynecology, Peking Union Medical College Hospital, Chinese Academy of Medical Sciences and Peking Union Medical College, Beijing, China

Endometrial intraepithelial neoplasia (EIN), also known as endometrial atypical hyperplasia $(E A H)$ is believed to be the precursor lesion of endometrioid endometrial carcinoma (EEC). Many genetic factors play important roles in the process of carcinogenesis, however, the key genetic alterations from dysplasia to endometrial cancer remains poorly understood. Germline mutations in Lynch syndrome genes are associated with hereditary endometrial carcinoma. The role of other cancer susceptibility genes is unclear. The aim of this study was to investigate the genomic alterations of premalignant endometrial lesion and EEC, and to determine the prevalence of cancer predisposition gene mutations in an unselected endometrial carcinoma patient cohort. Here, we applied a comprehensive cancer gene panel (363 cancer-related genes) to capture the exomes of cancer-related genes. Samples were collected from 79 patients with EEC and 36 patients with EIN. Our results demonstrate that EIN harbors most of the driver events reported in EEC and for the first time we reported a high frequency of the amplification of VEGFB gene in endometrial cancer. Moreover, we identified four novel candidate cancer-associated genes (CTCF, ARHGAP35, NF1, and KDR) which may be crucial in the carcinogenesis of EEC. In addition, we identified 2 patients who had a deleterious germline mutation in Lynch syndrome genes (MLH1 and MLH2), and another 8 patients harbored germline mutations of 6 non-Lynch syndrome genes (MUTYH, GALNT12, POLE, MPL, ATM, and ERCC4) which may be associated with endometrial cancer. Larger series will have to be investigated to assess the risks and the proportion of endometrial cancers attributable to other genes.

Keywords: endometrioid endometrial cancer, endometrial intraepithelial neoplasia, next generation sequencing, predisposing genes, carcinogenesis 


\section{INTRODUCTION}

Endometrial cancer (EC) is the most frequent gynecological cancer in developed countries, globally affecting more than 380,000 new women each year (1). In recent years, with the rapid development of the economy in China, people's living habits and dietary structure have undergone great changes. Accompanied by the increase of metabolic diseases, the incidence of endometrial cancer is gradually increasing. In China, endometrial cancer ranks second among gynecological cancers in its incidence, with approximately 63,400 new cases diagnosed in 2015 (2). Approximately 80\% of all endometrial carcinomas are of endometrioid endometrial carcinoma (EEC), which are associated with long stimulation of estrogen without antagonism of progestogen and have a favorable prognosis (3). The genetic landscape of EEC has been characterized in 2013 by TCGA, which reveals frequent mutations in PTEN, CTNNB1, PIK3CA, ARID1A, KRAS, and novel mutations in the SWI/SNF chromatin remodeling complex gene ARID5B (4).

Endometrial intraepithelial neoplasia (EIN) is considered to be the precursor lesion of EEC. Approximately $20 \%$ of the EIN will progress into EC, but the molecular pathogenesis underlying this progression is poorly explored. Thus, there is a demand for comprehensive exploration of the underlying genetic ordering events. Previous studies demonstrate that EIN share genetic similarity with EEC such as microsatellite instability(MSI) and somatic mutations of PTEN, PIK3CA, CTNNB1, and KRAS (5-9). In addition, EIN also have notable different specific mutations, which suggests activation of specific pathways by different mutational mechanisms. However, due to the small sample size and limited detected genes, researchers did not find any statistically significant genetic differences between EIN and EEC and the difference of molecular profiles between them still remains obscure (8). Which molecular variations play crucial role in initiating the progression from dysplasia to carcinoma has not been determined. Hence, it is important to explore the molecular features of EIN and EEC, which may also be valuable for the clinical management.

Lynch syndrome (LS) is a hereditary cancer syndrome caused by germline mutations in the mismatch repair (MMR) genes (MLH1, MSH2, MSH6, and PMS2), which has been regarded as the most common hereditary cancer syndrome (10). Patients of LS have an increased lifetime risk of colorectal, endometrial, ovarian, and many other cancers (11). In addition to LS, other hereditary syndromes characterized by deleterious germline mutations in other genes (such as PTEN, MUTYH) are less common and rarely investigated in Chinese population (12).

Although previous studies detailed the patterns of somatic alterations across primary endometrial tumors. To our knowledge, no comparative study has reported on molecular profiles of premalignant endometrial lesions and endometrioid endometrial cancer in Chinese population. Such information would be helpful in understanding the underlying genetic ordering events in endometrial cancer progression. Herein, we performed high-depth targeted sequencing to detect the mutational status in 363 cancer-related genes from 115 paired fresh-frozen tissues and whole blood samples of patients within EIN and EEC.

\section{MATERIALS AND METHODS}

\section{Patients and Samples}

We obtained fresh-frozen tumor blocks and paired germline blood samples from 115 individuals (36 with EIN and 79 with EEC) who underwent surgery between September 2017 and September 2018 at Peking Union Medical College Hospital (PUMCH). Patients' characteristics are listed in Table 1. The diagnosis of cases was confirmed by at least 2 experienced gynecological pathologists. Tumor staging and grading were performed according to International Federation of Gynecology and Obstetrics (FIGO) 2014 standards. This study was approved by the Ethics Committee of PUMCH, Beijing, China (HS-1704). Written informed consent was obtained from all participants of this study at admission to PUMCH.

\section{DNA Isolation, Library Construction, and Amplification, Targeted Capture, and Illumina-Based Sequencing}

Genetic testing was performed after pathological diagnosis of EIN and EEC. Genomic DNA was extracted from fresh frozen tissue (somatic) and the matched blood sample (germline) using the DNeasy Blood \& Tissue Kit (Qiagen, Hilden, Germany) according to the manufacturer's instructions. DNA was purified by AxyPrep ${ }^{\mathrm{TM}}$ Mag Tissue-Blood genomic DNA Kit (Axygen, America). And its quantification was measured by the Qubit dsDNA HS Assay Kit (Life Technologies, Eugene, OR). All purified DNA samples are judged to be of high quality (concentration > $3.4 \mathrm{ng} / \mathrm{ul}$ ) for mutation analysis. Fifty nanograms of genomic DNA was fragmented randomly into fragments which size range from 200 to 300 bp. Fragmented DNA was added with barcode and adaptors using polymerase chain reaction (PCR) reagents, the quality

TABLE 1 | Patient demographics in the high-throughput sequencing experiment.

\begin{tabular}{llc}
\hline Characteristics & Classification & \\
\hline Age, median (range) & EIN & $36(20-61)$ \\
Pathological classification & EC & $57(26-80)$ \\
& EIN & 36 \\
FIGO staging of EC & G2 EEC & 40 \\
& G3 EEC & 24 \\
& IA & 15 \\
& IB & 61 \\
II & 4 \\
IIIA & 3 \\
IIIIB & 4 \\
IIIC & 2 \\
IV & 4 \\
\end{tabular}

EIN, endometrial intraepithelial neoplasia; EEC, endometrioid endometrial carcinoma; EC, endometrial cancer. 
of PCR products was checked. The products were used for library construction and follow-up exon capture. Captured fragments were subsequently purified, amplified, ligated, and circularized by NimbleGen SeqCap EZ Hybridization and Wash Kit (Roche NimbleGen, Madison, WI). Finally, high-throughput sequencing of library products was performed on NextSeq CN500 (BerryGenomics, China).

\section{SNV and INDEL Calling}

After the sequencing, the FASTQ file was used for alignment and variant calling. To filter poor-quality reads, flexbar V2.4 software was used to process the raw read data files by removing the sequence of the original reads data and low-quality sequenced bases. The retained sequencing reads were then aligned to the reference human genome (NCBI Human Genome Buid37, hg19) using BWA (Burrows-Wheeler Aligner, version 0.5.9) software. SAM tools (Utilities for the Sequence Alignment/Map format, version 1.57) were used to integrate the matching information. GATK (Genome-Analysis Tool Kit, version 3.6), a widely used genetic variants discovery tool, was applied to identify single nucleotide variations (SNVs) and INDELs according to the sequence alignment results. Other databases and tools were also used to annotate identified genetic variants, including Variant Tools Version 3.0 (https://vatlab.github.io/vat-docs/), ANNOVAR Version 3.5a (http://annovar.openbioinformatics. org/en/latest/), dbSNP database (https://www.ncbi.nlm.nih.gov/ SNP/), Clinvar (https://www.ncbi.nlm.nih.gov/clinvar/), Cosmic V86 (https://cancer.sanger.ac.uk/cosmic/). Tumor mutational burden (TMB) was determined by the number of SNVs (depth $>150 \mathrm{X}$ and allele frequency $\geq 0.03$ ) which was detected on NGS (interrogating $\mathrm{Mb}$ of the genome), and the value was extrapolated to the whole exome using a validated algorithm.

\section{Determination of Potential Driver Mutations}

A bioinformatic algorithm called MutSigCV (13) was to predict the probability that an individual gene functions as a driver gene in the initiation and progression of endometrial cancer. As for several specific genes not listed in the MutSigCV based database, Tumor Suppressor and Oncogene (TUSON) Explorer (14), a computational method that analyses the patterns of mutational signatures in tumors and predicts the likelihood that any individual gene functions as a tumor suppressor (TSG) or oncogene (OG) was applied.

\section{Copy Number Analysis}

Copy number variation (CNV) was called from read counts by algorithm written by Berry Genomics. The details of this algorithm are as follows: After obtaining the sequencing data alignment information, the gene is divided into multiple regions and the average sequencing depth of each region is counted. Then, the sequencing depth of each region and its corresponding normal tissue sample is compared and the LRR value (LRR $=\log _{2} \frac{\text { sequencing depth of the tumor sample }}{\text { sequencing depth of the normal tissue sample }}$ ) of each region is calculated. According to LRR value, determine whether each region is amplified (LRR $>0.35$ ) or deleted (LRR $<0.5$ ). Finally, the proportion of the number of amplified and deleted regions of each gene is counted, and the type (amplification or deletion) which accounts for more than $70 \%$ is considered as the copy number variation type of the gene. Functional annotation of the differentially mutated genes was performed by use of the Gene Set Enrichment Analysis (GSEA) (http:// software.broadinstitute.org/gsea/index.jsp).

\section{Statistical Analysis}

The Pearson's $\chi 2$-test or the Fisher exact test was used for a comparison of frequencies between two groups. All tests were two sided, and $P<0.05$ was considered statistically significant. All statistical analyses were performed with SPSS version 22.0 software (SPSS Inc., Chicago, IL, USA).

\section{RESULTS}

\section{Overview of Sequencing Data}

DNA from all samples was successfully extracted and amplified by multiplex PCR, which was all qualified for library construction and next-generation sequencing. The genomic profiles of EIN and EEC were analyzed by a designed panel which targeted 363 cancer-related genes (Supplementary Figure 1). On average, we obtained 27 million 50-nt single reads for tumor samples and 23 million reads for matched blood samples. For the targeted regions, the average depth of coverage was $1690 \times$ and $2389 \times$ for tumor and matched blood samples, respectively. The average coverage rate of the target region was $99.35 \%$ (Supplementary Table 1).

Sequencing data for all samples meets somatic mutation analysis requirements. After variant filtration, we identified a total of 1,803 variants in 310 genes, including 1,620 single-nucleotide variants (SNVs) and 183 small scale insertions/deletions (indels) (Supplementary Table 2). All EC cases were identified at least one alteration among the 363 genes screened, while 5 cases of EIN had no somatic mutation identified. Across the entire dataset, the median TMB was 5.07 mutations/Mb, with a range of $0-233.93$ mutations/Mb. For all ECCs, the median TMB was 5.49 mutations/Mb, with a range of 0.84-233.93 mutations/Mb. For all EIN, the median TMB was 2.955 mutations $/ \mathrm{Mb}$, with a range of $0-16.05$ mutations $/ \mathrm{Mb}$. Patients with EEC had a significantly higher median TMB than patients with EIN (5.49 vs. $2.955, p=0.000$ ) (Figure 1A).

There were five highly mutated patients (median 173 per tumor, range from 141 to 277 ) and the remaining 110 patients were non-highly mutated (median 6 mutations per tumor; range $0-48)$. We further investigated and found that hypermutated phenotype group could be attributed to a disturbed DNA repair system resulted from the mutated genes involving in DNA damage repair pathway such as POLE, POLQ and mismatch repair (MMR) genes (MSH2, MSH6, MLH1, MLH3, PMS1, and PMS2). Details of the mutated genes in DNA damage repair pathway were shown in Supplementary Table 3. To distinguish the actual difference of TMB between EEC and EIN, we excluded samples with mutations in DNA damage repair pathway and the result showed the median TMB of two groups had no significant difference (5.07 vs. $3.80, p=0.294$ ) (Figure 1B). 


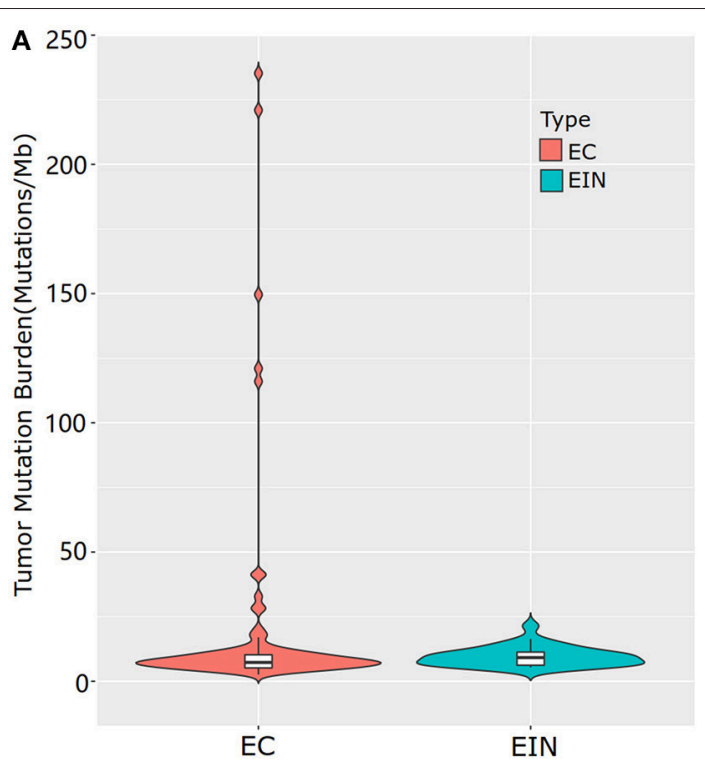

B

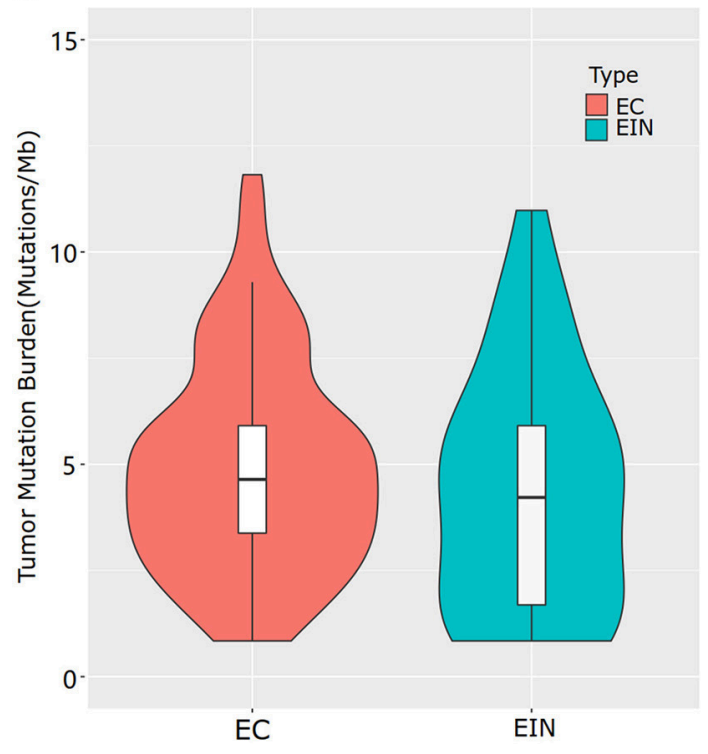

FIGURE 1 | Tumor mutational burden levels of EEC and EIN. (A) comparison of TMB which included all samples of EEC. (B) comparison of TMB which excluded samples of EEC with mutations in genes involved in DNA damage repair pathway. EIN, endometrial intraepithelial neoplasia; EEC, endometrioid endometrial carcinoma.

\section{Genomic Profiles of Premalignant Endometrial Lesions and Endometrial Cancer}

\section{Mutated Genes and Predicted Driver Genes}

In all cases, the most frequently altered genes were PTEN (53.9\%), PIK3CA (46.1\%), CTNNB1 (29.6\%), PIK3R1 (29.6\%), ARID1A (28.7\%), KRAS (11.5\%), CTCF (13.0\%), FGFR2 (12.2\%), and ARID5B (11.3\%) (Figure 2). PTEN, PIK3R1, and ARID1A alterations were significantly more commonly occurred in cases of endometrial cancer than in EIN ( $p$ all $<0.05$ ) (Table 2). We find that 4 cases (11.1\%) with EIN and 24 cases (30.4\%) with EEC have concurrent mutations of PTEN and PIK3CA $(P=0.046)$. Simultaneous mutations of PTEN and PIK3R1 genes were identified in 23 cases, and the occurrence rate in EC is higher than EIN with no significant difference $(24.1 \%$ vs. $11.1 \%, P=0.175)$. CTNNB1 mutations were identified in $29.6 \%$ (34/115) samples, with 38 non-synonymous variants and 1 non-frameshift deletion detected. $79.5 \%$ (31/39) variants occurred in exon 3. T41I (c.122C $>\mathrm{T}$ ) is the most frequently recurrent variant followed by S37F $(\mathrm{c} .110 \mathrm{C}>\mathrm{T})$ and R93M (c.278G $>\mathrm{T})$. According to the JAXClinical Knowledgebase annotation, 31 variants can lead to an accumulation of CTNNB1 expression products which can activate cell proliferation. In addition, pathological examination revealed that all samples harboring CTNNB1 mutation were well or moderate differentiated. KRAS mutations were identified in $15.7 \%(18 / 115)$ cases, and $88.9 \%$ mutations occurred in exon 2. Of three recurrent variants, G12V (c.35G > T) is the most commonly (38.9\%) occurred followed by G12D (c.35G > A, $27.8 \%)$ and $\mathrm{Q} 61 \mathrm{H}$ (c.183A > C, 11.1\%). ARID1A mutations were identified in $27.8 \%(32 / 115)$ samples, and a total of 49 variants were detected. 42.9\% (21/49) mutations occurred in exon 20. But only R1989X was recorded in present database which was a pathogenic variant.

According to the MutSigCV analysis result, 44 genes were predicted to be genetic drivers of endometrial cancer $(P<0.01$, Figure 2). And the top 9 highly mutated genes (PTEN, PIK3CA, PIK3R1, CTNNB1, ARID1A, KRAS, CTCF, FGFR2, ARID5B) together with TP53 and FOXA2 had stronger tendency to be tumor driver genes compared to other genes $\left(P<10^{-7}\right.$, Figure 2). To be noticed that, 4 genes (ARHGAP35, KMT2D, KMT2B, KMT2C) were not included in the MutSigCV database, but ranks high in TUSON Explorer (top 100 TSGs), which was shown in Figure 2 and Supplementary Table 2.

\section{Copy Number Alteration Data}

Of 115 samples, copy number alterations (CNAs) occurred in $48(41.7 \%)$ samples (Figure 3). The overall CNA burden was relatively low (median 2 CNAs per sample, range 0-37). The overwhelming majority of CNAs were amplification of genes, and the amplification of VEGFB gene was the most frequent CNA which was presented in 20/115 samples of which $19 / 20$ were endometrial cancer (95\%) and 1/20 were EIN (5\%). Indeed, earlier reports reported this gene was associated with tumorigenesis (15).

\section{Differentially Mutated Genes}

Of the frequently mutated genes, the mutation frequencies of 7 genes (PTEN, PIK3R1, ARID1A, KRAS, CTCF, ARHGAP35, and KDR) had significant difference between EIN and EEC groups ( $P$ all < 0.05). Besides, mutations of CTCF, ARHGAP35, NF1, KDR, and TP53 were only detected in EEC cases (Table 2; Figure 2). Of these genes, we identified 15 somatic CTCF mutations in EEC samples, and the majority mutations resulted from frameshift (33.3\%) and non-sense (46.7\%) mutations. And a mutational hotspot p.T204fs was identified in 4 patients. As for mutations of ARHGAP35, 50\% mutations resulted from missense mutations, 


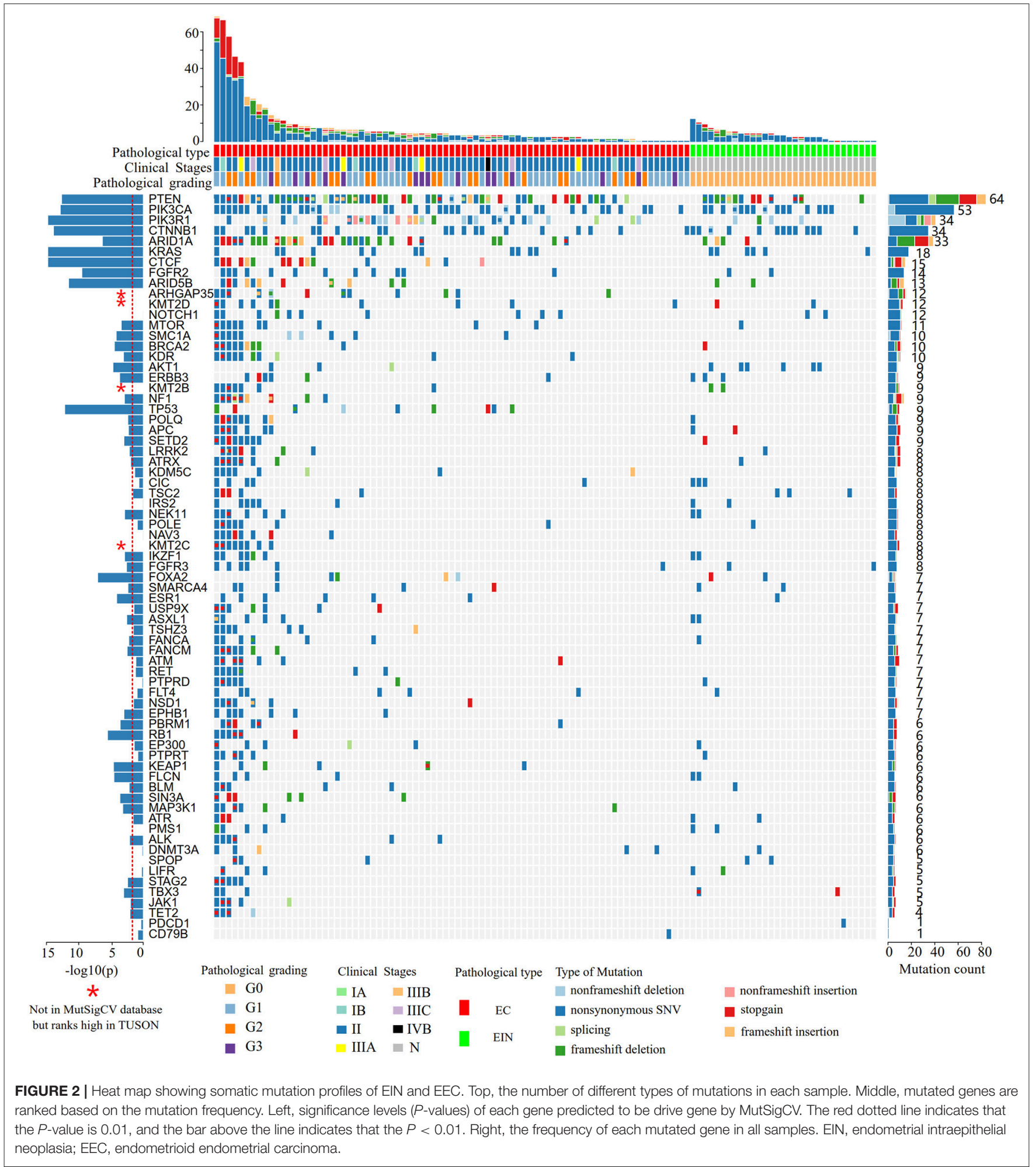

$33.3 \%$ resulted from frameshift mutations and the remaining (20\%) were non-sense and non-frameshift deletion mutations. Nine patients with EEC harbored NF1 mutations, of which there were two recurrent mutational spots (p.R2450 and p.L2639I). The TP53 mutation frequency in our study was $7.8 \%$ and 2 mutations occurred in patients with well-differentiated EEC. A recurrent mutational spot (p.A379V) in KDR gene were identified in 2 patients.

Moreover, we identified the pathways which top 10 differentially mutated genes clustered in (Table 3). The key 
TABLE 2 | The highly mutated genes (mutation frequency $\geq 9$ ) in all samples.

\begin{tabular}{|c|c|c|c|c|c|c|}
\hline Gene symbol & Total prevalence & $\begin{array}{c}\text { MutSigCV } \\
P \text {-value }\end{array}$ & Pathway & EEC group & EIN group & $P$-value \\
\hline PTEN & $64 / 115$ (53.9\%) & $5.88 \times 10^{-15}$ & PI (3)K pathway & $51 / 79$ & $13 / 36$ & 0.008 \\
\hline PIK3CA & $53 / 115$ (46.1\%) & $3.44 \times 10^{-15}$ & PI (3)K pathway & $37 / 79$ & $16 / 36$ & 0.811 \\
\hline CTNNB1 & $34 / 115$ (29.6\%) & $2.22 \times 10^{-16}$ & RTK/RAS/ $\beta$-catenin & $22 / 79$ & $12 / 36$ & 0.607 \\
\hline PIK3R1 & $34 / 115$ (29.6\%) & $2.22 \times 10^{-17}$ & PI (3)K pathway & $30 / 79$ & $4 / 36$ & 0.004 \\
\hline ARID1A & $33 / 115$ (28.7\%) & $8.78 \times 10^{-8}$ & SNF/SWI & $29 / 79$ & $4 / 36$ & 0.007 \\
\hline KRAS & 18/115 (15.7\%) & $2.22 \times 10^{-17}$ & RTK/RAS/ $\beta$-catenin & $12 / 79$ & $6 / 36$ & 0.840 \\
\hline CTCF & $15 / 115$ (12.2\%) & $2.22 \times 10^{-17}$ & - & $15 / 79$ & $0 / 36$ & 0.003 \\
\hline FGFR2 & $14 / 115(12.2 \%)$ & $2.17 \times 10^{-11}$ & RTK/RAS/ $\beta$-catenin & $12 / 79$ & $2 / 36$ & 0.247 \\
\hline ARID5B & 13/115 (11.3\%) & $9.96 \times 10^{-14}$ & - & $11 / 79$ & $2 / 36$ & 0.340 \\
\hline NOTCH1 & $12 / 115(10.4 \%)$ & 0.997 & Notch signaling pathway & $9 / 79$ & $3 / 36$ & 0.866 \\
\hline ARHGAP35 & 12/115 (10.4\%) & 1 & - & $12 / 79$ & $0 / 36$ & 0.009 \\
\hline KMT2D & $12 / 115$ (10.4\%) & 1 & - & $10 / 79$ & $2 / 36$ & 0.409 \\
\hline MTOR & $11 / 115(9.6 \%)$ & $1.79 \times 10^{-4}$ & PI (3)K pathway & $10 / 79$ & $1 / 36$ & 0.184 \\
\hline SMC1A & $10 / 115(8.7 \%)$ & $2.31 \times 10^{-5}$ & - & $9 / 79$ & $1 / 36$ & 0.168 \\
\hline BRCA2 & $10 / 115(8.7 \%)$ & $1.12 \times 10^{-5}$ & - & $9 / 79$ & $1 / 36$ & 0.168 \\
\hline KDR & $10 / 115(8.7 \%)$ & $4.44 \times 10^{-4}$ & - & $10 / 79$ & $0 / 36$ & 0.030 \\
\hline AKT1 & $9 / 115$ (7.8\%) & $6.37 \times 10^{-6}$ & PI (3)K pathway & $3 / 79$ & $6 / 36$ & 0.045 \\
\hline APC & $9 / 115$ (7.8\%) & $3.28 \times 10^{-3}$ & - & $8 / 79$ & $1 / 36$ & 0.324 \\
\hline KMT2B & $9 / 115(7.8 \%)$ & 1 & - & $7 / 79$ & $2 / 36$ & 0.812 \\
\hline ERBB3 & $9 / 115$ (7.8\%) & $9.30 \times 10^{-5}$ & - & $7 / 79$ & $2 / 36$ & 0.812 \\
\hline NF1 & $9 / 115$ (7.8\%) & $6.56 \times 10^{-4}$ & - & $9 / 79$ & $0 / 36$ & 0.055 \\
\hline TP53 & $9 / 115$ (7.8\%) & $2.07 \times 10^{-14}$ & P53 pathway & $9 / 79$ & $0 / 36$ & 0.055 \\
\hline POLQ & $9 / 115(7.8 \%)$ & $2.58 \times 10^{-3}$ & - & $8 / 79$ & $1 / 36$ & 0.324 \\
\hline
\end{tabular}

Statistically significant values are shown in bold.

mutated genes of GO enrichment analysis were Intracellular signal transduction, Positive regulation of biosynthetic process, Regulation of organelle organization and Regulation of cell matrix adhesion. As shown in Table 3, the most significantly mutated genes enriched KEGG pathways were Endometrial cancer, Focal adhesion, Glioma, Melanoma, Small cell lung cancer and Prostate cancer.

\section{Germline Mutations}

Table 4 summarizes age at diagnosis, tumor histology, and family history for patients with deleterious germline mutation in Lynch and non-Lynch syndrome genes. There were no germline mutations detected in EIN, whereas 10 patients with EEC (10/115) harbored 10 pathogenic or likely pathogenic germline mutations in 8 different genes. Notably, identification of a germline MMR mutation was detected in 2 patients with EEC $(2 / 79,2.5 \%)$. And both of them had also undergone immunohistochemistry expression of mismatch repair proteins for evaluation of possible Lynch Syndrome after surgery. On patient had loss of MLH1 and PMS2 expression, and the other had loss of MSH2 and MSH6 expression. Eight patients were identified with germline mutations in nonLynch syndrome genes. Two endometrial cancer patients were identified with MUTYH homozygous c.934-2A>G or heterozygous c.G467A (p.W156X) mutation. And one patient harbored a pathogenic variant of MPL (c.981-1G>C).
Potentially pathogenic variants were found in five Cancer Gene Census germline genes: GALNT12, POLE, MPL, ATM, and ERCC4.

\section{DISCUSSION}

Regarding molecular evolution of EIN and EEC, EIN is the result of a series of mutations involving multiple genes, including tumor suppressor genes, oncogenes, mismatch repair, and genes that control cell growth, differentiation and apoptosis (16). Not all precursor lesions of endometrial cancer can progress to carcinoma, therefore, it is of great importance to distinguish which patients with EIN will develop to EC, and this will be helpful for clinicians to tailor therapeutic interventions. Previous studies have demonstrated that dysplasia and endometrial cancer share several aberrant characteristics with each other. However, the ordering events from dysplasia to carcinoma and which molecular alteration in precursor lesions can predict cancer progression remain unclear. Recently, Russo et al. performed next-generation sequencing on paired EIN and EEC from a series of hysterectomy specimens, and their result suggested the progression from EIN to EEC was not a linear accumulation of mutational events. However, based on a limited sample size, no difference was observed in mutational burden, CNA burden, or specific mutational genes between EEC and EIN (8). In this study, we screened for mutations in 363 genes in endometrial cancer 


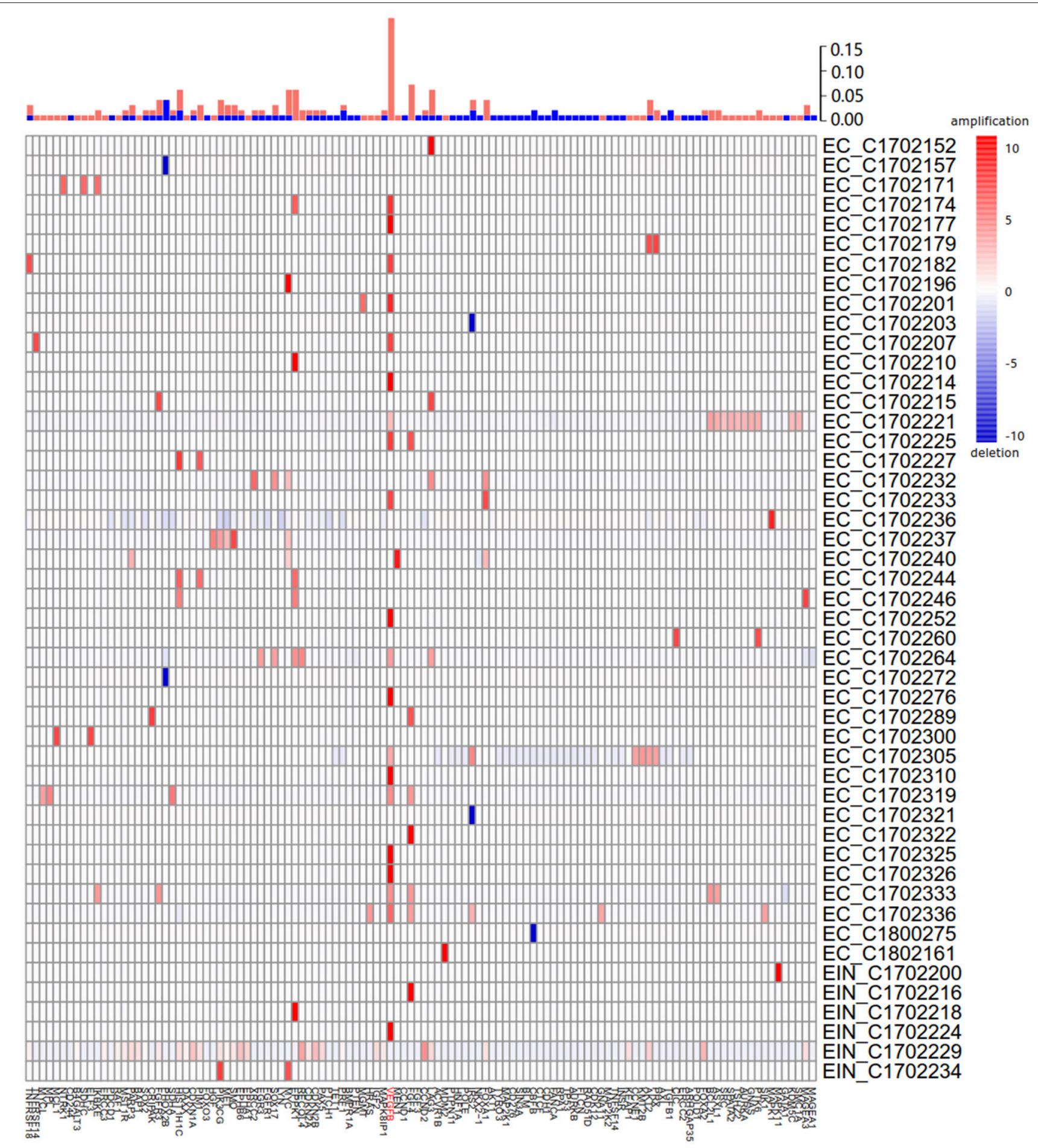

FIGURE 3 | Summary of all copy number aberrations in 48 samples. Upper bar, frequency of amplifications and deletions. Middle bar, heatmap of copy number aberration profiles of 48 samples. Amplified and deleted genomic regions are shown in red and blue, respectively. The high frequency of amplification of VEGFB is easily noticeable.

and its precursor lesions using high-throughput sequencing. We believe that this study will shed new light on fundamental aspects for understanding the molecular pathogenesis of endometrial cancer and may aid in the development of new targeted therapies.

\section{Major Signaling Pathway}

Alterations in $\mathrm{PI} 3 \mathrm{~K} / \mathrm{AKT} / \mathrm{mTOR}$ signaling pathway are very common in endometrial carcinoma and its precursor lesions $(17,18)$. Of the genes involved in this pathway examined in 
TABLE 3 | Top 10 significantly mutated GO terms and KEGG pathways.

\begin{tabular}{|c|c|c|c|c|}
\hline Term & Description & Mutated genes & $\boldsymbol{P}$-value & FDR \\
\hline \multicolumn{5}{|l|}{ GO TERM } \\
\hline GO:1902531 & Intracellular signal transduction & PIK3R1, PTEN, AKT1, TP53, KDR, NF1, ARHGAP35 & $7.80 \times 10^{-11}$ & $3.60 \times 10^{-7}$ \\
\hline GO:0009889 & Positive regulation of biosynthetic process & PIK3R1, PTEN, AKT1, TP53, KDR, NF1, CTCF, ARID1A & $2.34 \times 10^{-10}$ & $4.36 \times 10^{-7}$ \\
\hline GO:0033043 & Regulation of organelle organization & PIK3R1, PTEN, AKT1, TP53, KDR, CTCF & $8.01 \times 10^{-10}$ & $6.17 \times 10^{-7}$ \\
\hline GO:0007160 & Regulation of cell matrix adhesion & PIK3R1, PTEN, KDR, NF1 & $2.86 \times 10^{-9}$ & $1.32 \times 10^{-6}$ \\
\hline \multicolumn{5}{|c|}{ KEGG PATHWAY } \\
\hline hsa05213 & Endometrial cancer & PIK3R1, PTEN, AKT1, TP53 & $3.04 \times 10^{-10}$ & $4.36 \times 10^{-7}$ \\
\hline hsa04510 & Focal adhesion & PIK3R1, PTEN, AKT1, KDR, ARHGAP35 & $3.77 \times 10^{-10}$ & $4.36 \times 10^{-7}$ \\
\hline hsa05214 & Glioma & PIK3R1, PTEN, AKT1, TP53 & $7.60 \times 10^{-10}$ & $6.17 \times 10^{-7}$ \\
\hline hsa05218 & Melanoma & PIK3R1, PTEN, AKT1, TP53 & $1.09 \times 10^{-9}$ & $7.20 \times 10^{-7}$ \\
\hline hsa05222 & Small cell lung cancer & PIK3R1, PTEN, AKT1, TP53 & $2.16 \times 10^{-9}$ & $1.25 \times 10^{-6}$ \\
\hline hsa05215 & Prostate cancer & PIK3R1, PTEN, AKT1, TP53 & $2.86 \times 10^{-9}$ & $1.32 \times 10^{-6}$ \\
\hline
\end{tabular}

FDR, false discovery rate.

TABLE 4 | Ten patients with germline mutations in 363 genes detected using NGS of 115 paired samples.

\begin{tabular}{|c|c|c|c|c|c|c|}
\hline Gene & Base change/Variant effect & Mutant type & ClinVar/COSMIC & Age at diagnosis & Histology & Family history \\
\hline MUTYH & c.934-2A>G & hom & Pathogenic & 34 & G1EEC & None \\
\hline MLH1 & c.885-1_893delGTTAGAAAT & hom & Pathogenic & 28 & G3 EEC & CRC-FDR, SDR \\
\hline $\mathrm{MSH} 2$ & c.C1183T (p.Q395X) & hom & Pathogenic & 49 & G1 EEC & None \\
\hline GALNT12 & c.G5A (p.W2X) & hom & Likely pathogenic & 26 & G1 EEC & None \\
\hline POLE & c.4430delG (p.S1477Tfs) & het & Likely pathogenic & 63 & G1 EEC & CRC-FDR \\
\hline MPL & c.313_316delTTTC (p.F105Rfs) & het & Likely pathogenic & 54 & G1 EEC & None \\
\hline ATM & c.C8494T (p.R2832C) & het & Likely pathogenic & 61 & G2 EEC & None \\
\hline MUTYH & c.G467A (p.W156X) & het & Pathogenic & 51 & G2 EEC & None \\
\hline ERCC4 & c.1536dupA (p.G513Rfs) & het & Likely pathogenic & 57 & G3 EEC & None \\
\hline MPL & c. $981-1 G>C$ & het & Pathogenic & 45 & G1 EEC & None \\
\hline
\end{tabular}

CRC, colorectal cancer; FDR, first-degree relative; SDR, second-degree relative; het, heterozygous; hom, homozygous; EEC, endometrioid endometrial carcinoma.

our study, PTEN, PIK3CA, and PIK3R1 all mutated frequently, which was similar to the TCGA study on endometrial carcinoma (4). In an early study with small sample size, researchers explored the status of the PIK3CA gene and its association with PTEN mutations in EIN and EC and found a significantly lower frequency of PIK3CA mutations in EIN (5). While in our study, the frequency of PIK3CA mutations between EIN and EEC is comparable $(52.7 \%$ vs. $39.4 \%, P=0.811)$. However, a significantly lower frequency of PTEN and PIK3R1 mutations in EIN were identified. As a transforming oncogene, mutation of PIK3CA gene is an alternative to PTEN mutation, which also leads to an increase in $\mathrm{PI} 3 \mathrm{~K} / \mathrm{AKT} / \mathrm{mTOR}$ pathway activity and are important for the invasive potential (19). Previous studies have showed frequent coexistence of PIK3CA and PTEN mutations (17). In our study, 24.3\% (28/115) patients have simultaneous mutations in these two genes, and $85.7 \%$ $(24 / 28)$ simultaneous mutations occurred in EC. These results all suggested PIK3CA mutations may have a synergic effect with PTEN inactivation in the development of endometrial tumors. As for the associated survival outcome, the literature suggests that mutations of PI3K pathway may be associated with worse clinical outcomes (20-22).
$\mathrm{WNT} / \beta$-catenin signaling pathway is the second most frequently activated pathway in EEC (4), which plays a pivotal role in numerous cellular processes such as cell proliferation, differentiation, and maintenance of pluripotency (23). Mutated Wnt pathway components are causative to multiple growthrelated pathologies and to cancer (24). $\beta$-catenin which is encoded by CTNNB1 gene, is the key downstream effector of this signaling pathway (25). CTNNB1 mutations have been detected in endometrial hyperplasia, suggesting that these mutations occur in the early stages of the neoplastic process. Researchers have found that CTNNB1 mutations can identify a subset of low grade, early stage endometrial cancer patients (26), which was also proved by our study. Mutation in exon 3 of CTNNB1 gene is classically associated with translocation of the $\beta$-catenin protein from the membrane to the nucleus and activation of $\mathrm{Wnt} / \beta$ catenin signaling $(27,28)$. Jeong et al. (29) found exon 3 deletion of the CTNNB1 gene in a murine model led to upregulation of the Wnt/beta-catenin pathway and the development of endometrial hyperplasia. In present study, $79.5 \%$ mutations of CTNNB1 occurred in exon 3, and according to the gene annotation database, most variants lead to an accumulation of CTNNB1 expression products ( $\beta$-catenin) which can activate cell 
proliferation. These features make $\beta$-catenin (more specifically, a change in its overall expression and/or subcellular localization) an attractive potential marker for EIN and EEC.

The RAS-RAF-MEK-ERK pathway is frequently mutated in human cancers. Of three RAS isoforms, KRAS is the most frequently mutated in human cancers (30), and KRAS activation has been proved as an early oncogenic event in endometrial carcinogenesis (31), which correlates with mucinous differentiation in cancers (32). Although no mucinous component has been detected in our samples, we still identified $15.7 \%$ patients had KRAS mutations, and nearly all the mutations occur in codon 12 or 13 of exon 2 of the gene. The hotspot mutations can lead to a mutant oncoprotein, which can decrease its GTPase activity and increase activation of downstream signaling, stimulating neoplastic transformation (33).

In addition to the above mentioned several pivotal signaling pathways involved in transforming endometrial cells to the primary carcinogenesis and metastasis (34), our study for the first time demonstrated a high frequency of the amplification of VEGFB gene. VEGFB is a member of VEGF family, which regulates the formation of blood vessels and involves in endothelial cell physiology (35). It promotes cancer metastasis through the remodeling of tumor microvasculature, and previous study showed high expression levels of VEGF-B in patients with lung squamous cell carcinoma and melanoma correlated with poor survival (15). Targeting VEGFB may be an important therapeutic approach for cancer metastasis. So far, there is no research on the effect of VEGFB on the prognosis of endometrial cancer, and future studies are required to elucidate it. Moreover, we identified the pathways differentially mutated genes between EEC and EIN involved in, such as intracellular signal transduction, positive regulation of biosynthetic process, regulation of organelle organization and regulation of cell matrix adhesion pathways. These pathways may be involved in the multistep development of endometrial cancer. In the future, clinical trials of drugs for endometrial cancer that target these pathways may be carried out.

\section{Novel Candidate Genes in Malignant Transformation}

One of the major findings of our study is that we found different genetic features between EEC and its precursor lesions. Of highly mutated genes detected, ARHGAP35, CTCF, NF1, KDR, and TP53 mutations were only detected in EC cases. Except TP53, other genes have rarely been explored in endometrial cancer before. ARHGAP35 (also known as p190RHOGAP) encoding glucocorticoid receptor DNA-binding factor-1 is a cancer associated gene with a mutation spectrum suggestive of a tumor suppressor function $(36,37)$. Activation of ARHGAP35 causes RhoA inactivation and inhibits cell invasion (38), while its inactivation could play a role in tumor development $(39,40)$. Deletion of chromosomal region encompassing ARHGAP gene has been described in solid tumors (41). Abnormal expression of ARHGAP35 in colorectal cancer patients was associated with poor survival (42). However, inactivating mutation status of ARHGAP35 remains unknown in endometrial carcinoma.
Our study for the first time reported that ARHGAP 35 gene mutation in endometrial cancer is a distinguishable mutation characterization from its precursor lesions. According to our MutSigCV result, ARHGAP35 is not predicted to be a true cancer driver gene, but a pan-cancer study suggested it may contribute to oncogenesis of endometrial cancer (43). Future research will investigate its expression and clinical significance in endometrial cancer.

CTCF encoding a highly conserved 11-zinc finger DNA binding protein is mutated in about $15 \%$ of endometrial cancer (4). Previous study suggested that CTCF is a tumor suppressor and it can regulate the expression of various cancer-related genes (44-46). Loss of CTCF binding can induce epigenetic silencing of tumor suppressor loci or lead to activation of oncogenes. Marshall et al. (47) identified the pro-tumorigenic roles of CTCF mutations in endometrial cancer, and also showed that CTCF haploinsufficiency was associated with poor prognosis. In this study, we only identified CTCF mutations in EEC, and confirmed a recurrent frameshift defect (p.T204Nfs).

The NF1 gene encodes a protein called neurofibromin that is known to function as a tumor suppressor (48). Currently, a genome-wide association study(GWAS) conducted by O'Mara and Glubb (49) identified 9 new susceptibility loci for endometrial cancer, and one loci is NF1 (17q11.2) which encodes a negative regulator of RAS-mediated signal transduction (48).KDR also known as VEGFR, encodes a receptor of vascular endothelial growth factor and has a pivotal role in promoting cancer angiogenesis (50). Research showed its mutation was associated with pancreatic cancer prognosis (51). However, mutations of this gene have rarely been explored in endometrial cancer. According to our MutSigCV result, it was also a putative cancer-associated driver gene.

Based on the above findings, we surmise that these newly discovered four genes (CTCF, ARHGAP35, NF1, and KDR) may correlate with malignant transformation.

\section{Germline Mutations}

Recently, advanced in the next-generation sequencing technology has begun to reshape the field of cancer genetics. Genetic testing not only helps in the diagnosis of cancer and the selection of targeted drugs, but the identification of causative genetic mutations helps predict cancer risk and even achieve cancer prevention through intensive screening and surgical prophylaxis (52). More than 100 cancer predisposition genes have been identified to date $(53,54)$, while the relationship between susceptibility gene and relevant tumor types still needs further investigation. Most recently, a study which included 10,389 cases across 33 different cancers identified pathogenic or likely pathogenic variants in $8 \%$ of all patients (55). However, the genetic architecture of endometrial cancer susceptibility is not well-known. Knowledge of genetic susceptibility to endometrial cancer mainly comes from several hereditary cancer-predisposing syndromes.

Lynch syndrome (LS) is described as an inherited predisposition to colorectal cancer and other cancers, including EC and OC (56). It is caused by autosomal dominant mutations in DNA mismatch repair genes (MLH1, MSH2, MSH6, and 
PMS2), and women with Lynch syndrome have a cumulative lifetime risk of endometrial cancer of $20-70 \%$ (57). In our cohort, $8.7 \%$ of patients were found to have pathogenic or likely pathogenic germline mutations. Two of them were carriers of deleterious mutation of MMR genes. One of the patients had a family history of cancers that met the Amsterdam criteria II. The other patient had no family history of any cancer, and had a concurrent stage IA ovarian endometrioid adenocarcinoma.

In our study, 8 patients were identified with pathogenic or likely pathogenic germline mutations in non-Lynch syndrome genes (MUTYH, GALNT 12, POLE, MPL, ATM, and ERCC4), which indicated that there may be other genes outside of Lynch syndrome associated with endometrial cancer. In the future, a germline multi-gene panel targeted on endometrial cancer may be applied to identify additional actionable mutations in endometrial cancer. Germline biallelic inactivation of MUTYH represents a familial cancer syndrome, and patients of biallelic MUTYH mutation carriers have an increased risk of developing colorectal cancer $(58,59)$. At present there is little information about the role of MUTYH in other types of cancer. Biallelic MUTYH mutations have been found in patients affected with endometrial carcinoma, and a few reports indicated a possible relationship with endometrial cancer (60). In our cohort, we identified 2 patients carried pathogenic mutations of MUTYH gene, and only one was bi-allelic which was considered deleterious.

In addition to MUTYH, there were another five genes which were identified with germline variants in patients with endometrial cancer. However, there are no reports on the relationship between MPL, GALNT12 and endometrial cancer to date. Interestingly, the other three germline mutated genes (ATM, ERCC4, and POLE) are all involved in the DNA damage repair pathway (61), which are key factors in maintain genomic integrity and stability. Germline mutations in ATM gene are thought to contribute to breast cancer susceptibility (62). And previous study suggested germline mutations affecting the exonuclease domain of POLE predispose to CRC and endometrial cancer (63). Whether these additional gene mutation carriers are susceptible to endometrial cancer should be explored in future studies.

\section{CONCLUSION}

Our study, for the first time, portrayed the mutational spectra of both EEC and its precursor lesions in Chinese population. According to our data, EC bears a higher mutational burden than its precursor lesion which attribute to a disturbed DNA repair system resulting from the mutated genes in DNA damage repair pathway. EIN harbored most of the significantly mutated genes which were also prevalent in EEC. We identified several cancer driver genes and defined the pathway involved in the oncogenesis of endometrial cancer. We also identified four novel candidate genes (CTCF, ARHGAP35, NF1, and KDR) which may correlate with malignant transformation of dysplasia. Our findings in germline mutations also suggest that except for Lynch syndrome genes, there are other non-lynch syndrome genes which may associate with endometrial cancer. Larger series will have to be investigated to assess the risks and the proportion of endometrial cancers attributable to other genes. In conclusion, our work represents the beginning step of investigating the genetic relationship between EEC and its precursor lesions. In the future, integrated multi-omics analysis will shed further light on the development of EEC.

\section{DATASET WITH ACCESSION NUMBER}

The raw sequence data reported in this paper have been deposited in the Genome Sequence Archive (Genomics, Proteomics \& Bioinformatics 2017) in BIG Data Center (Nucleic Acids Res 2018), Beijing Institute of Genomics (BIG), Chinese Academy of Sciences, under accession number HRA000033. URL: http://bigd. big.ac.cn/gsa-human/s/U6AiGcb4.

\section{AUTHOR CONTRIBUTIONS}

MY and J-XY conceived and designed the research. YW and $\mathrm{J}$-XY performed the research. YW is responsible for data analysis and drafted the manuscript. MY and J-XY discussed the results and amended the manuscript. D-YC, YZ, and H-MZ supervised the research. ZY and KS provisioned useful suggestions in table and figure preparation. All authors read and approved the final version of the manuscript.

\section{FUNDING}

This work was supported by grants from the Chinese Academy of Medical Sciences Initiative for Innovative Medicine (CAMS2017-I2M-1-002).

\section{ACKNOWLEDGMENTS}

The authors thank all of the faculty, nurses, and staff at Department of Obstetrics \&Gynecology in Peking Union Medical College Hospital for the excellent care they provide to patients. We are also grateful to the colleges at Department of Pathology for their support in pathology review. The authors also sincerely thank all the patients and their family members for their contribution to this research effort. We are also grateful for the help provided by Qingzheng Zhang and Lingling Pang from Berry Oncology Co., Ltd. on bioinformatics analysis.

\section{SUPPLEMENTARY MATERIAL}

The Supplementary Material for this article can be found online at: https://www.frontiersin.org/articles/10.3389/fonc. 2019.00123/full\#supplementary-material 


\section{REFERENCES}

1. Bray F, Ferlay J, Soerjomataram I, Siegel RL, Torre LA, Jemal A. Global cancer statistics 2018: GLOBOCAN estimates of incidence and mortality worldwide for 36 cancers in 185 countries. CA Cancer J Clin. (2018) 68:394-424. doi: $10.3322 /$ caac. 21492

2. Chen W, Zheng R, Baade PD, Zhang S, Zeng H, Bray F, et al. Cancer statistics in China, 2015. CA Cancer J Clin. (2016) 66:115-32. doi: 10.3322/caac.21338

3. Amant F, Moerman P, Neven P, Timmerman D, Van Limbergen E, Vergote I. Endometrial cancer. Lancet. (2005) 366:491-505. doi: 10.1016/S0140-6736(05)67063-8

4. Kandoth C, Schultz N, Cherniack AD, Akbani R, Liu Y, Shen H, et al. Integrated genomic characterization of endometrial carcinoma. Nature. (2013) 497:67-73. doi: 10.1038/nature12113

5. Hayes MP, Wang H, Espinal-Witter R, Douglas W, Solomon GJ, Baker SJ, et al. PIK3CA and PTEN mutations in uterine endometrioid carcinoma and complex atypical hyperplasia. Clin Cancer Res. (2006) 12:5932-5. doi: 10.1158/1078-0432.CCR-06-1375

6. Nieminen TT, Gylling A, Abdel-Rahman WM, Nuorva K, Aarnio M, Renkonen-Sinisalo L, et al. Molecular analysis of endometrial tumorigenesis: importance of complex hyperplasia regardless of atypia. Clin Cancer Res. (2009) 15:5772-83. doi: 10.1158/1078-0432.CCR-09-0506

7. Vierkoetter KR, Kagami LA, Ahn HJ, Shimizu DM, Terada KY. Loss of mismatch repair protein expression in unselected endometrial adenocarcinoma precursor lesions. Int J Gynecol Cancer. (2016) 26:228-32. doi: 10.1097/IGC.0000000000000606

8. Russo M, Broach J, Sheldon K, Houser KR, Liu DJ, Kesterson J, et al. Clonal evolution in paired endometrial intraepithelial neoplasia/atypical hyperplasia and endometrioid adenocarcinoma. Hum Pathol. (2017) 67:6977. doi: 10.1016/j.humpath.2017.07.003

9. Van Der Putten LJM, Van Hoof R, Tops BBJ, Snijders M, Van Den Berg-Van Erp SH, Van Der Wurff A, et al. Molecular profiles of benign and (pre)malignant endometrial lesions. Carcinogenesis. (2017) 38:329-35. doi: $10.1093 /$ carcin/bgx008

10. Win AK, Jenkins MA, Dowty JG, Antoniou AC, Lee A, Giles GG, et al. Prevalence and penetrance of major genes and polygenes for colorectal cancer. Cancer Epidemiol Biomarkers Prev. (2017) 26:404-12. doi: 10.1158/1055-9965.EPI-16-0693

11. Vasen HF, Blanco I, Aktan-Collan K, Gopie JP, Alonso A, Aretz S, et al. Revised guidelines for the clinical management of Lynch syndrome (HNPCC): recommendations by a group of European experts. Gut. (2013) 62:812-23. doi: 10.1136/gutjnl-2012-304356

12. Pilarski R, Burt R, Kohlman W, Pho L, Shannon KM, Swisher E. Cowden syndrome and the PTEN hamartoma tumor syndrome: systematic review and revised diagnostic criteria. J Natl Cancer Inst. (2013) 105:1607-16. doi: $10.1093 /$ jnci/djt277

13. Lawrence MS, Stojanov P, Polak P, Kryukov GV, Cibulskis K, Sivachenko A, et al. Mutational heterogeneity in cancer and the search for new cancerassociated genes. Nature. (2013) 499:214-8. doi: 10.1038/nature12213

14. Davoli T, Xu AW, Mengwasser KE, Sack LM, Yoon JC, Park PJ, et al. Cumulative haploinsufficiency and triplosensitivity drive aneuploidy patterns and shape the cancer genome. Cell. (2013) 155:948-62. doi: 10.1016/j.cell.2013.10.011

15. Yang X, Zhang Y, Hosaka K, Andersson P, Wang J, Tholander F, et al. VEGF-B promotes cancer metastasis through a VEGF-A-independent mechanism and serves as a marker of poor prognosis for cancer patients. Proc Natl Acad Sci USA. (2015) 112:E2900-9. doi: 10.1073/pnas.15 03500112

16. Steinbakk A, Gudlaugsson E, Aasprong OG, Skaland I, Malpica A, Feng W, et al. Molecular biomarkers in endometrial hyperplasias predict cancer progression. Am J Obstet Gynecol. (2011) 204:357 e1-12. doi: 10.1016/j.ajog.2010.12.007

17. Velasco A, Bussaglia E, Pallares J, Dolcet X, Llobet D, Encinas M, et al. PIK3CA gene mutations in endometrial carcinoma: correlation with PTEN and K-RAS alterations. Hum Pathol. (2006) 37:1465-72. doi: 10.1016/j.humpath.2006.05.007

18. Strickland AL, Rivera G, Lucas E, John G, Cuevas I, Castrillon DH. PI3K Pathway effectors pAKT and FOXO1 as novel markers of endometrioid intraepithelial neoplasia. Int J Gynecol Pathol. (2018). doi: 10.1097/PGP.0000000000000549. [Epub ahead of print].

19. Eritja N, Yeramian A, Chen BJ, Llobet-Navas D, Ortega E, Colas E, et al. Endometrial carcinoma: specific targeted pathways. Adv Exp Med Biol. (2017) 943:149-207. doi: 10.1007/978-3-319-43139-0_6

20. Catasus L, Gallardo A, Cuatrecasas M, Prat J. PIK3CA mutations in the kinase domain (exon 20) of uterine endometrial adenocarcinomas are associated with adverse prognostic parameters. Mod Pathol. (2008) 21:131-9. doi: 10.1038/modpathol.3800992

21. Salvesen HB, Carter SL, Mannelqvist M, Dutt A, Getz G, Stefansson IM, et al. Integrated genomic profiling of endometrial carcinoma associates aggressive tumors with indicators of PI3 kinase activation. Proc Natl Acad Sci USA. (2009) 106:4834-9. doi: 10.1073/pnas.0806514106

22. Nout RA, Bosse T, Creutzberg CL, Jurgenliemk-Schulz IM, Jobsen JJ, Lutgens LC, et al. Improved risk assessment of endometrial cancer by combined analysis of MSI, PI3K-AKT, Wnt/beta-catenin and P53 pathway activation. Gynecol Oncol. (2012) 126:466-73. doi: 10.1016/j.ygyno.2012.05.012

23. Dravid G, Ye Z, Hammond H, Chen G, Pyle A, Donovan P, et al. Defining the role of Wnt/beta-catenin signaling in the survival, proliferation, and selfrenewal of human embryonic stem cells. Stem Cells. (2005) 23:1489-501. doi: 10.1634/stemcells.2005-0034

24. Nusse R, Clevers H. Wnt/beta-catenin signaling, disease, and emerging therapeutic modalities. Cell. (2017) 169:985-99. doi: 10.1016/ j.cell.2017.05.016

25. Gumbiner BM. Signal transduction of beta-catenin. Curr Opin Cell Biol. (1995) 7:634-40. doi: 10.1016/0955-0674(95)80104-9

26. Kurnit KC, Kim GN, Fellman BM, Urbauer DL, Mills GB, Zhang W, et al. CTNNB1 (beta-catenin) mutation identifies low grade, early stage endometrial cancer patients at increased risk of recurrence. Mod Pathol. (2017) 30:1032-41. doi: 10.1038/modpathol.2017.15

27. Morin PJ, Sparks AB, Korinek V, Barker N, Clevers H, Vogelstein $\mathrm{B}$, et al. Activation of beta-catenin-Tcf signaling in colon cancer by mutations in beta-catenin or APC. Science. (1997) 275:1787-90. doi: $10.1126 /$ science.275.5307.1787

28. Kim G, Kurnit KC, Djordjevic B, Singh C, Munsell MF, Wang WL, et al. Nuclear $\beta$-catenin localization and mutation of the CTNNB1 gene: a context-dependent association. Mod Pathol. (2018) 31:1553-9. doi: 10.1038/s41379-018-0080-0

29. Jeong JW, Lee HS, Franco HL, Broaddus RR, Taketo MM, Tsai SY, et al. beta-catenin mediates glandular formation and dysregulation of beta-catenin induces hyperplasia formation in the murine uterus. Oncogene. (2009) 28:3140. doi: $10.1038 /$ onc. 2008.363

30. Schubbert S, Shannon K, Bollag G. Hyperactive Ras in developmental disorders and cancer. Nat Rev Cancer. (2007) 7:295-308. doi: 10.1038/nrc2109

31. Berg A, Hoivik EA, Mjos S, Holst F, Werner HM, Tangen IL, et al. Molecular profiling of endometrial carcinoma precursor, primary and metastatic lesions suggests different targets for treatment in obese compared to non-obese patients. Oncotarget. (2015) 6:1327-39. doi: 10.18632/oncotarget.2675

32. Alomari A, Abi-Raad R, Buza N, Hui P. Frequent KRAS mutation in complex mucinous epithelial lesions of the endometrium. Mod Pathol. (2014) 27:67580. doi: 10.1038/modpathol.2013.186

33. Hunter JC, Manandhar A, Carrasco MA, Gurbani D, Gondi S, Westover KD. Biochemical and structural analysis of common cancerassociated KRAS mutations. Mol Cancer Res. (2015) 13:1325-35. doi: 10.1158/1541-7786.MCR-15-0203

34. Ma X, Ma CX, Wang J. Endometrial carcinogenesis and molecular signaling pathways. Am J Mol Biol. (2014) 4:134-49. doi: 10.4236/ajmb.2014.43015

35. Olofsson B, Pajusola K, Kaipainen A, Von Euler G, Joukov V, Saksela O, et al. Vascular endothelial growth factor B, a novel growth factor for endothelial cells. Proc Natl Acad Sci USA. (1996) 93:2576-81. doi: 10.1073/pnas.93.6.2576

36. Ludwig K, Parsons SJ. The tumor suppressor, p190RhoGAP, differentially initiates apoptosis and confers docetaxel sensitivity to breast cancer cells. Genes Cancer. (2011) 2:20-30. doi: 10.1177/1947601911402680

37. Frank SR, Kollmann CP, Luong P, Galli GG, Zou L. p190 RhoGAP promotes contact inhibition in epithelial cells by repressing YAP activity. J Cell Biol. (2018) 217:jcb.201710058. doi: 10.1083/jcb.201710058

38. Bartolome RA, Diaz-Martinez M, Colo GP, Arellano-Sanchez N, TorresAyuso P, Kleinovink JW, et al. A Blk-p190RhoGAP signaling module 
downstream of activated Galpha13 functionally opposes CXCL12-stimulated RhoA activation and cell invasion. Cell Signal. (2014) 26:2551-61. doi: 10.1016/j.cellsig.2014.07.008

39. Wang DZ, Nur EKMS, Tikoo A, Montague W, Maruta H. The GTPase and Rho GAP domains of p190, a tumor suppressor protein that binds the $\mathrm{M}(\mathrm{r})$ 120,000 Ras GAP, independently function as anti-Ras tumor suppressors. Cancer Res. (1997) 57:2478-84.

40. Sun Q, Cibas ES, Huang H, Hodgson L, Overholtzer M. Induction of entosis by epithelial cadherin expression. Cell Res. (2014) 24:1288-98. doi: $10.1038 /$ cr.2014.137

41. Tikoo A, Czekay S, Viars C, White S, Heath JK, Arden K, et al. p190$\mathrm{A}$, a human tumor suppressor gene, maps to the chromosomal region $19 q 13.3$ that is reportedly deleted in some gliomas. Gene. (2000) 257:23-31. doi: 10.1016/S0378-1119(00)00387-5

42. Li L, Li YM, Zhou P, Wang XS, Wang GY, Zhao XH, et al. Abnormal expression of p190RhoGAP in colorectal cancer patients with poor survival. Am J Transl Res. (2016) 8:4405-14.

43. Lawrence MS, Stojanov P, Mermel CH, Robinson JT, Garraway LA, Golub TR, et al. Discovery and saturation analysis of cancer genes across 21 tumour types. Nature. (2014) 505:495-501. doi: 10.1038/nature12912

44. Lobanenkov VV, Nicolas RH, Adler VV, Paterson H, Klenova EM, Polotskaja $\mathrm{AV}$, et al. A novel sequence-specific DNA binding protein which interacts with three regularly spaced direct repeats of the CCCTC-motif in the 5'-flanking sequence of the chicken c-myc gene. Oncogene. (1990) 5:1743-53.

45. Rasko JE, Klenova EM, Leon J, Filippova GN, Loukinov DI, Vatolin S, et al. Cell growth inhibition by the multifunctional multivalent zinc-finger factor CTCF. Cancer Res. (2001) 61:6002-7.

46. Soto-Reyes E, Recillas-Targa F. Epigenetic regulation of the human p53 gene promoter by the CTCF transcription factor in transformed cell lines. Oncogene. (2010) 29:2217-27. doi: 10.1038/onc.2009.509

47. Marshall AD, Bailey CG, Champ K, Vellozzi M, O'young P, Metierre C, et al. CTCF genetic alterations in endometrial carcinoma are pro-tumorigenic. Oncogene. (2017) 36:4100-10. doi: 10.1038/onc.2017.25

48. Rad E, Tee AR. Neurofibromatosis type 1: fundamental insights into cell signalling and cancer. Semin Cell Dev Biol. (2016) 52:39-46. doi: 10.1016/j.semcdb.2016.02.007

49. O'mara TA, Glubb DM. Identification of nine new susceptibility loci for endometrial cancer. Nat Commun. (2018) 9:3166. doi: 10.1038/s41467-018-05427-7

50. Elsayed NMY, Serya RAT, Tolba MF, Ahmed M, Barakat K, Abou El Ella DA, et al. Design, synthesis, biological evaluation and dynamics simulation of indazole derivatives with antiangiogenic and antiproliferative anticancer activity. Bioorg Chem. (2018) 82:340-59. doi: 10.1016/j.bioorg.2018.10.071

51. Cheng H, Liu C, Jiang J, Luo G, Lu Y, Jin K, et al. Analysis of ctDNA to predict prognosis and monitor treatment responses in metastatic pancreatic cancer patients. Int J Cancer. (2017) 140:2344-50. doi: 10.1002/ijc.30650

52. Hall MJ, Obeid EI, Schwartz SC, Mantia-Smaldone G, Forman AD, Daly MB. Genetic testing for hereditary cancer predisposition: BRCA1/2, Lynch syndrome, and beyond. Gynecol Oncol. (2016) 140:565-74. doi: 10.1016/j.ygyno.2016.01.019
53. Lu C, Xie M, Wendl MC, Wang J, Mclellan MD, Leiserson MD, et al. Patterns and functional implications of rare germline variants across 12 cancer types. Nat Commun. (2015) 6:10086. doi: 10.1038/ncomms10086

54. Zhang J, Walsh MF, Wu G, Edmonson MN, Gruber TA, Easton J, et al. Germline mutations in predisposition genes in pediatric cancer. N Engl J Med. (2015) 373:2336-46. doi: 10.1056/NEJMoa1508054

55. Huang KL, Mashl RJ, Wu Y, Ritter DI, Wang J, Oh C, et al. Pathogenic germline variants in 10,389 adult cancers. Cell. (2018) 173:355-70.e314. doi: 10.1016/j.cell.2018.03.039

56. Dunlop MG, Farrington SM, Carothers AD, Wyllie AH, Sharp L, Burn J, et al. Cancer risk associated with germline DNA mismatch repair gene mutations. Hum Mol Genet. (1997) 6:105-10. doi: 10.1093/hmg/6.1.105

57. Stoffel E, Mukherjee B, Raymond VM, Tayob N, Kastrinos F, Sparr J, et al. Calculation of risk of colorectal and endometrial cancer among patients with Lynch syndrome. Gastroenterology. (2009) 137:1621-7. doi: 10.1053/j.gastro.2009.07.039

58. Farrington SM, Tenesa A, Barnetson R, Wiltshire A, Prendergast J, Porteous $\mathrm{M}$, et al. Germline susceptibility to colorectal cancer due to base-excision repair gene defects. Am J Hum Genet. (2005) 77:112-9. doi: 10.1086/ 431213

59. Kairupan CF, Meldrum CJ, Crooks R, Milward EA, Spigelman AD, Burgess B, et al. Mutation analysis of the MYH gene in an Australian series of colorectal polyposis patients with or without germline APC mutations. Int J Cancer. (2005) 116:73-7. doi: 10.1002/ijc.20983

60. Tricarico R, Bet P, Ciambotti B, Di Gregorio C, Gatteschi B, Gismondi V, et al. Endometrial cancer and somatic G>T KRAS transversion in patients with constitutional MUTYH biallelic mutations. Cancer Lett. (2009) 274:266-70. doi: 10.1016/j.canlet.2008.09.022

61. Chan SH, Lim WK, Ishak NDB, Li ST, Goh WL, Tan GS, et al. Germline mutations in cancer predisposition genes are frequent in sporadic sarcomas. Sci Rep. (2017) 7:10660. doi: 10.1038/s41598-017-10333-x

62. Estiar MA, Mehdipour P. ATM in breast and brain tumors: a comprehensive review. Cancer Biol Med. (2018) 15:210-27. doi: 10.20892/j.issn.2095-3941.2018.0022

63. Church DN, Briggs SE, Palles C, Domingo E, Kearsey SJ, Grimes $\mathrm{JM}$, et al. DNA polymerase epsilon and delta exonuclease domain mutations in endometrial cancer. Hum Mol Genet. (2013) 22:2820-8. doi: $10.1093 / \mathrm{hmg} / \mathrm{ddt} 131$

Conflict of Interest Statement: The authors declare that the research was conducted in the absence of any commercial or financial relationships that could be construed as a potential conflict of interest.

Copyright (c) 2019 Wang, Yu, Yang, Cao, Zhang, Zhou, Yuan and Shen. This is an open-access article distributed under the terms of the Creative Commons Attribution License (CC BY). The use, distribution or reproduction in other forums is permitted, provided the original author(s) and the copyright owner(s) are credited and that the original publication in this journal is cited, in accordance with accepted academic practice. No use, distribution or reproduction is permitted which does not comply with these terms. 